\title{
La práctica de la milpa, el ch'ulel y el maíz como elementos articuladores de la cosmovisión sobre la naturaleza entre los tzeltales de Tenejapa en los Altos de Chiapas
}

\section{The Milpa Practices, Ch'ulel and Maize as Integrative Elements of Nature Cosmology among the Tzeltal from Tenejapa in the highlands of Chiapas}

\author{
RenZo D’Alessandro \\ Centro de Investigaciones y Estudios Superiores en Antropología Social, Unidad Sureste \\ Alma Amalia GonzÁlez \\ Centro de Investigaciones Multidisciplinarias sobre Chiapas y la Frontera Sur, UNAM
}

\begin{abstract}
Resumen: Este artículo presenta los resultados del trabajo etnográfico realizado en comunidades tzeltales de Tenejapa en los Altos de Chiapas donde se analizan, a partir de las prácticas agrícolas bajo el sistema de milpa, la relación humano-naturaleza. Se propone una descripción etnográfica para comprender mejor la construcción de los territorios tzeltales desde una aproximación teórica que aborda la relación cultura-naturaleza desde la ecología simbólica de Descola. Asimismo, se retoman algunas de las aportaciones sobre la concepción de la naturaleza entre los mayas para exponer la relevancia de sus mitos, rituales y prácticas agrícolas como elementos conciliatorios entre la cultura y la naturaleza, y como expresión de la apropiación del territorio.
\end{abstract}

Palabras clave: milpa, maíz, ch’ulel, tzeltal, cosmología, ontologías indígenas.

ABSTRACT: This article presents the results of ethnographic work in Tzeltal communities in the municipality of Tenejapa in Los Altos of Chiapas, where we analyzed the relationship between human and nature based on the agricultural practices of the "milpa" system. It offers an ethnographic description trying to understand the construction of Tzeltal territories with a theoretical approach that seeks to understand the relationship between culture and nature from the symbolic ecology proposed by Descola. We also draw up several studies to analyze the perspectives of nature among the Maya in order to expose the relevance of their myths, rituals and agricultural practices merging culture and nature and as an expression of territory appropriation.

KEYwords: milpa, maíz, ch’ulel, tzeltal, cosmology, indigenous ontology.

Résumé: Cet article présente les résultats de travaux ethnographiques dans les communautés tzeltal dans la municipalité de Tenejapa dans Los Altos du Chiapas où, la relation entre l'homme et la nature peut s'analyser à partir des pratiques agri- 
coles du système "milpa". On propose Une description ethnographique que essai de comprendre la construction des territoires tzeltal avec une approche théorique qui cherche à comprendre la relation entre la culture et la nature selon l'écologie symbolique proposé par Descola. On a également repris certaines contributions sur la conception de la nature chez les Mayas pour exposer la pertinence de leurs mythes, les rites et les pratiques agricoles comme des éléments de conciliation entre la culture et la nature, et comme une expression de l'appropriation du territoire.

Moтs clé: milpa, maíz, ch'ulel, tzeltal, cosmologie, ontologie autochtone.

RECEPCIÓN: 30 de noviembre de 2015.

ACEPTACIÓN: $1^{\circ}$ de marzo de 2016.

DOI: 10.19130/iifl.ecm.2017.50.768.

\section{Introducción}

Las relaciones simbólicas y concepciones cosmogónicas de los mayas han sido ampliamente estudiadas desde diferentes perspectivas, grosso modo: una visión histórica y más general sobre "lo maya" desde el análisis de la literatura, los códices, el ethos y sus conocimientos etnobotánicos (De la Garza, 1975, 1984, 2012; Ruz, 1998, 2004; Sotelo, 1988; Florescano, 2000; Barrera-Bassols y Toledo, 2005; Ford y Nigh, 2015, por nombrar sólo algunos estudiosos del tema). Otra perspectiva, de tipo más local, es aquella que aparece en el margen de cierta moda de los estudios antropológicos del indigenismo sobre los mayas alteños de Chiapas (Holland, 1963; Guiteras, 1965; Villa Rojas, 2003; Hermitte, 1970; Pozas, 1977, entre otros). Finalmente, una corriente basada en los estudios antropológicos que retoman lo cosmogónico en la región Altos desde una perspectiva contemporánea (Vogt, 1966, 1979; 1966; Köhler, 1995; Medina, 1991; Pitarch, 1996, 2003; Breton, Monod y Ruz, 2003; Page, 2005; Estrada, 2009, entre otros).

La revista Estudios de Cultura Maya publicó un artículo de Adriana Estrada (2009) que proponía una forma de análisis de la tradición oral de los mayas para exponer las relaciones entre los humanos y la naturaleza. La propuesta de la autora se fundamenta teóricamente en la compilación que realizaron Descola y Pálsson (2001) sobre naturaleza y sociedad, la cual discute ampliamente el dualismo impuesto en la naturaleza y la cultura, dentro de dos tradiciones de la antropología social que explican las relaciones cultura-naturaleza (materialismo cultural y estructuralismo simbólico). Estrada señala algunos puntos conflictivos: la artificialidad en la construcción de la naturaleza desde la ciencia (Latour en Estrada, 2009); los errores típicos al intentar romper el paradigma dualista entre naturaleza y cultura (simplificación conceptual, textualismo, explicar los procesos de objetivación), y la definición de los problemas ambientales. En corolario, la compilación propuesta (Descola y Pálsson, 2001) discute la necesidad de mantener dentro de la antropología el estudio de los sistemas de subjetivación cognitiva de la naturaleza y no volcarse al análisis de las teorías de la acción. Es decir 
que la lupa de las ciencias sociales regrese al estudio de las representaciones culturales de la naturaleza de los pueblos y no exclusivamente a las formas de organización social y su evolución dentro del paradigma productivista y cambiante de la globalización.

De ahí la importancia del texto de Estrada, ya que responde al llamado de Descola y Pálsson para rescatar las concepciones de cultura y naturaleza dentro del pensamiento maya yucateco, aportando reflexiones sobre las relaciones existentes entre la gama de seres, espacios y tiempos que habitan en la cosmovisión de los mayas. Dicho planteamiento resulta interesante y complementario a la aparición de la obra de Descola (2005), publicada tres años antes que el artículo de Estrada. En este sentido, nuestra propuesta aporta un enfoque de análisis que complementa algunas ideas sobre las representaciones de la naturaleza en la cultura maya y asimismo presenta un marco de análisis, donde la práctica agrícola de la milpa se considera como elemento cosmogónico complementario al relato oral.

\section{Cosmovisión y modos de control y uso de la naturaleza}

La cosmogonía, como apuntó Godelier (1984), es el elemento fundamental de la construcción de valores que reinan en las relaciones humanas con el medio ambiente. La cosmovisión refiere a lo humano en su relación con el mundo y el universo. A lo humano como su parte constitutiva y a los ecosistemas como el campo en el que se desarrollan las relaciones entre lo humano consigo mismo y con el cosmos. La cosmovisión, desde la perspectiva que aquí se presenta, contiene la visión de los miembros de un grupo en la cual se combinan de forma coherente sus nociones poniendo énfasis en la relación con el medio ambiente, a partir de su observación, prácticas (repertorios tecnológicos) y saberes propios, que se sintetiza en los ritos agrícolas (Broda, 1991). ${ }^{1}$ La cosmovisión es un acuerdo que regula las relaciones sociales e implica que los cambios socio-económicos se harán visibles en los agroecosistemas, tal y como lo plantea Lévi-Strauss (1995) al exponer que las comunidades hacen de forma recurrente una traducción de signos y símbolos de la naturaleza.

Cuando hablamos de cosmovisiones hablamos directamente de las relaciones sociales que son mediadas por la cultura, es decir, entre las formas en las que

\footnotetext{
${ }^{1}$ Para analizar al sistema cosmológico se realizó una revisión a las aproximaciones teóricas que profundizan la relación cultura-naturaleza, a fin de entender y describir el papel del maíz en la agricultura milpera desde una perspectiva simbólica ligada a los valores sociales de los campesinos indígenas tzeltales. Se realizó una revisión bibliográfica sobre los mitos, ritos y relatos de los mayas referentes al maíz, y que se contrastaron mediante la observación participante en rituales y ceremonias de las diferentes comunidades, así como en los relatos de los tzeltales recabados mediante entrevistas en un trabajo etnográfico realizado en cuatro comunidades aledañas a la cabecera municipal de Tenejapa de marzo a julio de 2013.
} 
las culturas y los ecosistemas se influyen mutuamente: relaciones entre cultura y naturaleza. Para este análisis nos referiremos a dichas interacciones como "cultura-naturaleza".

Descola (2011) retoma la construcción epistemológica de las relaciones cultura -naturaleza desde dos corrientes opuestas: el materialismo ecológico y la ecología cultural. ${ }^{2}$ En la primera, el principio es que las adaptaciones culturales establecidas de una comunidad determinada y su repertorio tecnológico son creadas para explotar los recursos en un medio ambiente específico. Al sugerir que hasta las tribus más ocultas del planeta — como los jíbaros amazónicos—son portadores en esencia del homo-economicus, el materialismo ecológico contiene nociones que lo relacionan con el utilitarismo de la naturaleza. ${ }^{3}$ Por otro lado, en la segunda, desde el estructuralismo simbólico de Lévi-Strauss (1995), se plantea que la traducción de signos y símbolos de la naturaleza proviene de la cultura y que es ésta la que construye la idea de la naturaleza y no a la inversa. ${ }^{4}$ La relación cultura-naturaleza, que se propone para este trabajo, refiere a las cosmovisiones y las construcciones simbólicas de las comunidades indígenas tzeltales que persisten en su relación con la naturaleza.

El kosmos desde la perspectiva de la etnoecología (Toledo, 1996; Barrera-Bassols y Toledo, 2005) es un sistema de creencias propio de los grupos indígenas en el que existen mecanismos de negociación para regular el manejo y uso de los recursos naturales a su disposición. Asimismo, se retoma la idea de ecocosmología (Arhem, 2001) entendida como un conjunto de conocimientos, normas y valores fundamentales para permitir la conectividad entre humanos y naturaleza.

La propuesta de kosmos y la de ecocosmología conforman un sistema cosmológico local entendido como la memoria colectiva de las dimensiones sociales, materiales y espirituales que se expresa en los elementos simbólicos utilizados para la negociación/conciliación/conectividad en las relaciones sociales organizadas con la naturaleza en un territorio o nicho ecológico determinado. Los mecanismos de negociación implican una significación semiótica que integra a lo humano con lo natural mediante rituales, festividades y mito-prácticas. El sistema cosmológico se expresa en un conjunto de conocimientos trasmitidos por medio de la tradición que le da una significación colectiva al entorno que rebasa la dimensión de

\footnotetext{
${ }^{2}$ En este artículo nos referiremos indistintamente al materialismo ecológico y a la ecología cultural, dado que ambos pertenecen a una línea del pensamiento donde la idea principal de cultura está determinada por las características del entorno, entendido éste como la naturaleza.

${ }^{3}$ El materialismo ecológico se basa en la teoría de la decisión racional proveniente de la doctrina económica neoliberal (Descola, 2011: 22).

${ }^{4}$ Desde la lógica de la escuela ecológica cultural las relaciones cultura-naturaleza son un proceso de adaptación, donde el ambiente geográfico moldea la vida social. El entorno ecológico es el factor constituyente de los valores míticos que explican las relaciones simbólicas de la cultura con la naturaleza. Descola utiliza un interesante ejemplo para explicar las diferencias entre materialismo ecológico y estructuralismo simbólico contrastando las posiciones de Harris y Lévi-Strauss sobre un mito de Nueva Guinea relacionado con la utilización de las almejas, que ambos antropólogos analizan con conclusiones totalmente diferentes (ver "La querelle des palourdes" en Descola, 2011: 13).
} 
lo productivo. Lo cosmológico no se trasmite en tanto que conocimiento técnico sino como un cuerpo semántico de representaciones culturales a través de la transmisión de historias y creencias que engloba al "nosotros" y lo diferencia de la "otredad". López Austin señala la diferencia entre lo práctico (entendido como los conocimientos ligados a la domesticación del espacio, los animales y las plantas) y lo mítico de la siguiente forma:

(...) el agricultor no se conformó con construir su propio ámbito [práctico]. ${ }^{5}$ Como lo habían hecho sus antepasados recolectores y cazadores, modeló el otro ámbito, el paralelo, invisible, sutil y maravilloso; pródigo a veces y avaro en otras; a veces amoroso y en otras terrible. Reprodujo en este ámbito su naturaleza psíquica y su nueva condición social. Se retrató en dioses que lo escuchaban, que se condolían ante sus súplicas, que le reclamaban los lazos de la reciprocidad. Para él y para el maíz tejió la tupida red que entrelaza ambos ámbitos y habló a los dioses y de los dioses con la palabra, con la música, con la danza, con la ofrenda, con el manejo de los colores y los volúmenes, con el llanto y los suspiros de esperanza (2003: 29).

El sistema cosmológico está ligado a personajes o deidades de la memoria colectiva a través de íconos, relatos orales y representaciones míticas (como un universo de ideas, anécdotas, vivencias e interpretaciones que está en constante recreación) que conforman una interacción entre la cultura y la naturaleza mediada por rituales de vinculación.

El análisis del sistema cosmológico remite a una forma de comprender las relaciones sociales con la naturaleza a través la significación que tiene el maíz para los tzeltales mediante la exploración de sus objetos, altares, rituales y rezos vinculados con la siembra, la milpa y el entorno. Los sistemas cosmológicos son las percepciones, representaciones y patrimonios comunes que forman un rasgo que permite identificar un núcleo unificador (López, 2001), el cual es una expresión de la memoria colectiva de un grupo social que se transmite y se ajusta de generación en generación.

El mito de origen constituye la base del cómo y porqué del origen de la sociedad y del mundo; va cambiando, ajustándose a las necesidades sociales (muchas veces para justificar el porqué de las cosas, para imponer prohibiciones o para transgredirlas) y se trasmite a través de la tradición oral y ritual (Meslin en Page, 2005). Siguiendo a Descola (2003), aquí se propone que la dialéctica culturanaturaleza puede entenderse desde las relaciones simbólicas y las concepciones de los tzeltales de Los Altos de Chiapas sobre el maíz, como un elemento de intermediación con una naturaleza sacralizada. ${ }^{6}$ Para ello, se seleccionaron algunos de los elementos simbólicos que se expresan en sistemas de valores, nociones y

${ }^{5}$ La referencia a lo práctico entre corchetes es nuestra.

${ }^{6}$ Como se sabe, los tzeltales (principalmente dentro del catolicismo tradicional) consideran a las montañas, las cuevas, los manantiales, las plantas y algunos árboles como sagrados. La sacralidad de los lugares ha llevado a considerar que es el lugar sagrado la unidad de construcción del territorio. Una vez identificado el lugar, la población se adscribe ahí para adorar a los dioses. 
prácticas, es decir, aspectos o dimensiones del medio social, material y espiritual del saber compartido que guían las relaciones sociales presentes en las representaciones de las comunidades tzeltales ubicadas en el tiempo, el espacio, la sociedad y el cosmos.

\section{La naturaleza y la cultura desde el utilitarismo ecológico}

$\mathrm{Al}$ analizar la cultura y sus formas de interacción con la naturaleza se corre el riesgo de caer en trampas epistemológicas. Muchos de los argumentos en lugar de dar luz sobre la complejidad que pretende analizarse, fraccionan la discusión hacia una perspectiva unidisciplinaria, que tiene su base en el enfoque utilitarista de la naturaleza y que tiende a generar conclusiones que guardan un sentido político de lo sustentable y de la sustentabilidad.

Para ilustrar lo anterior, se recurre a las últimas mediciones globales de biodiversidad vegetal que afirman que "nunca antes en la historia de la humanidad habían existido tantas especies vegetales como en la actualidad". ${ }^{7}$ Los científicos no reconocen a lo antrópico como causa de una erosión biogenética global, sino que, por el contrario, recurren a afirmaciones en las que gracias a lo humano hay mayor diversidad de plantas, minimizando el desplazamiento de los ecosistemas por la invasión de la agricultura industrial o las disrupciones asociadas a los asentamientos humanos. ${ }^{8}$ Se puede decir que, en algunos casos, quienes realizan ciencia, responden a intereses y metodologías adscritas a paradigmas políticos y económicos cuya influencia utilitarista es dominante. Pero ipor qué predominan las perspectivas utilitaristas en las relaciones cultura-natura?

Las tipologías que varios autores han utilizado en los análisis de las relaciones cultura-naturaleza ${ }^{9}$ no niegan la existencia de un proceso de aculturación en las poblaciones indígenas, que afecta la forma de interactuar entre ellas mismas y con la naturaleza.

Existen vastos estudios sobre el tema de la aculturación de las sociedades indígenas dentro del sistema de economía colonialista e industrializada. ${ }^{10}$ La hipótesis principal en torno a la aculturación es que ésta ha generado una mayor

${ }^{7}$ Cita de la conversación informal con el Dr. Christian Leclerc, quien trabaja en un proyecto de medición de la biodiversidad global (septiembre de 2012, Montpellier, Francia). Sobre su trabajo al respecto ver Leclerc y Coppens (2011) o consultar Ellis, Antill y Kreft (2012).

${ }^{8}$ Sobre este punto se pueden ver Hobbs, Higgs y Harris (2009) y Ellis (2011).

${ }^{9}$ Marx las definía como relaciones sociales en torno al control de los modos de producción; Lapierre (1968), a partir de una graduación de su poder político, como desarrollado, poco desarrollado o ausente, y Gadgil y Guha (1992), en torno a la evolución en los modos de utilización en los recursos y los cambios socio-económicos (Ellison, 2013).

10 Se recomienda la obra de Guillermo Bonfil Batalla (2005) o la de Mario Ruz (1994). En esta última se encuentra un ensayo de Marie Chamoux "La difusión de tecnologías entre los indígenas de México: una interpretación”, que narra la imposición, favoritismo y apropiación de ciertos cultivos a partir de la Colonia en México. 
destrucción de la diversidad ecológica en los territorios donde actualmente habitan poblaciones indígenas, ${ }^{11}$ quienes gracias a sus saberes y prácticas han creado nichos culturales que permiten la existencia de biodiversidad local (Boege, 2009).

Disertar sobre por qué los territorios donde se asientan las comunidades indígenas son nichos de biodiversidad se explica mediante una hipótesis etno-ecológica que expone el desplazamiento geográfico y cultural de las comunidades indígenas mediante el cambio de racionalidades, pasando de una, adaptada al ecosistema, a otra racionalidad dominante y destructiva (Toledo, 1996). Los pueblos originarios se han tenido que desplazar históricamente de zonas de planicie (en muchos casos subhúmedas) a otras de montaña con menor acceso geográfico y con climas que van de sub-templados a fríos. Su propia concepción del bienestar ha tenido por consiguiente un modelo de prácticas y saberes ligados a una explotación menos intensiva de la naturaleza, lo que ha permitido la existencia y refugio de otras especies y, por ende, una amplia gama de biodiversidad y agrodiversidad (Boege, 2009).

El discurso de la racionalidad dominante se basa en la idea de "modernización y progreso" como el único medio para alcanzar bienestar. En ese juego de modernización y progreso, el Estado ha impuesto un régimen técnico-productivista contradictorio a los saberes, prácticas y formas simbólicas de la relación culturanaturaleza entre las poblaciones originarias.

La coexistencia de ambas racionalidades en un mismo territorio ha creado diferentes problemas de índole cultural, ${ }^{12}$ que globalmente han sido analizados por la antropología del desarrollo (Bastide, 1976) como fenómenos de integración de las comunidades campesinas-indígenas dentro de las sociedades industriales. Se plantea que una sociedad tradicional comenzará a diluirse dentro de una sociedad urbana industrial cuando los sistemas que regulan las decisiones sobre sus saberes (sistemas económicos, de organización social y cosmogónica) se integren. Existen en México varios procesos de integración de la sociedad tradicional a la urbana contemporánea. Uno de los más citados es el que surgió en los años cuarenta, durante la integración de las comunidades campesinas indígenas a la agricultura tecnológica y química, denominado Revolución verde", ${ }^{13}$ y ocurrido

11 Boege (2009) menciona que estos pueblos son quienes resguardan la biodiversidad, haciendo énfasis en el caso de la diversidad de razas de maíces: yaqui, mayo, pima, guarijío, tepehuán, rarámuri, cora, nahua, huichol, purépecha, otomí, matlazinca, mazahua, tlapaneco, triqui, amuzgo, mixteco, zapoteco, chatino, chontal, huave, huasteco, totonaca, pame, chichimeca, popoloca, cuicateco, mazateco, chinanteco, zoque, tzotzil, tzeltal, chol, lacandón, chol, kanjobal, chuj, tojolabal, chontal y mame.

${ }^{12}$ A principios del siglo xix en México llegó a existir un equilibrio económico entre las sociedades mestizas e indígenas en la sierra totonaca de Puebla. Al equilibrio le siguió una respuesta cultural de las sociedades mestizas en la que se generaron parámetros clasistas que denigraban socialmente lo indígena. A la postre esto sirvió para justificar y legitimar el desplazamiento de los grupos totonacos y la apropiación de sus tierras bajas en Huehuetla (Ellison, 2013).

${ }^{13}$ Sobre ello habría que ver los trabajos de Ángel Palerm, Arturo Warman, Guillermo Bonfil Batalla, Cynthia Hewitt de Alcántara, Luisa Paré, Roger Bartra, Víctor Toledo y otros tantos etnógrafos y antropólogos que han identificado los procesos de cambios rurales e indígenas en el campo mexicano. 
en años recientes con la integración económica y comercial que ha implicado el Tratado de Libre Comercio de América del Norte.

La aculturación desde el modelo dominante es un fenómeno que tiende a la uniformización y homogeneización de las prácticas de las poblaciones tradicionales al interrumpir el proceso de transmisión y reproducción de su cosmovisión y saberes originarios, es decir, aquellos elementos que están asociados a la construcción de sus objetos culturales y la significación que guía su relación naturaleza-comunidad. La aculturación se entiende como "la interrupción del proceso de transmisión del aprendizaje a través del cual una generación de más edad reproduce en la generación más joven la adopción de objetos, modos de pensamiento y comportamiento tradicionales" (Harris, 2003: 167).

La aculturación ha sido clasificada como un proceso de "integración cultural", entendido éste como el mecanismo donde suceden las innovaciones culturales cuando permea la introducción o transmisión de tecnología en la estructura (Steward y Shimkin, 1961). Los niveles de integración sociocultural están condicionados por las relaciones sociales de influencia entre diferentes miembros de un grupo cultural. Al afectarse las relaciones sociales se afectan las relaciones de transacción entre los miembros de un grupo. Si bien los niveles de integración cultural a la larga se expresan en cambios socioeconómicos que definirán el grado de vínculo social y de identidad local en las relaciones cultura-natura, dichos cambios terminarán "afectando las reglas colectivas en las que descansan las formas de acuerdo implícitas y explícitas, y que se apoyan en el vínculo social o la normatividad social" (Ellison, 2013: 41).

El origen del cambio en el núcleo cultural de los pueblos indígenas impacta en los repertorios tecnológicos — muchos de estos presentes en las prácticas agrícolas- y que en realidad no son difundidos, sino impuestos por las sociedades dominantes (Bonfil, 1989). Entonces, los cambios en las formas de vínculo social deben considerarse el resultado de elementos culturales ajenos al grupo cultural analizado, ya que no ha sido éste el que los ha producido.

Generalmente se dice que dichos saberes originarios son, por premisa, menos utilitarios y degradantes con el medio-ambiente por provenir de un modelo de explotación de los recursos naturales que no es tan intensivo como el de la civilización industrializada (Jacorzynski, 2004). Esta aseveración nos lleva a cuestionar la pertinencia de analizar el grado de autonomía en los saberes y prácticas que tienen los pueblos indígenas ante la intromisión de tecnologías ajenas a su cosmovisión, pero también a la cuestión de si es posible analizar una práctica "originaria”, toda vez que ha sido culturalmente "integrada", mediante las imposiciones tecnológicas que no le son propias en cuanto a su autoría.

La reproducción de las formas de relación cultura-naturaleza no está asociada con el grado de adaptación de las innovaciones tecnológicas ni a su implementación, sino a su autoría. Prueba de ello es que las comunidades indígenas no son las inventoras de los insumos tecnológicos agroquímicos o de semillas "mejoradas" de la revolución verde, sino tan sólo sus usuarias entrenadas. Las formas 
campesinas e indígenas de producción actual pueden entonces ser consideradas o no como sustentables, ya sea por sus prácticas propias (como la roza-tumbaquema) o por ser los usuarios de insumos externos, pero en el análisis de la sustentabilidad sólo se les pueden imputar los efectos derivados de aquellas prácticas de las que son artífices o precursores. Se puede decir que la cosmovisión cultura-natura no está definida por la utilización de una serie de componentes socio-técnicos ajenos, sino por aquellos de las tecnologías propuestas dentro de su propia cosmovisión.

Este análisis conlleva entonces a la conclusión de que no se puede definir una cosmovisión como más sustentable que otra sin analizar los procesos de aculturación y de imposición implícitos en la utilización de ciertas tecnologías, por lo que se debe integrar la autoría de la práctica a los criterios que definen los paradigmas de lo sustentable. La delimitación de lo sustentable, en el caso de los pueblos indígenas tzeltales, no emerge únicamente de las reglas colectivas que definen su relación o de los símbolos que se expresen aún a pesar de la preaculturación, sino de las tecnologías que ellos mismos desarrollan y aplican en su contacto con la naturaleza.

Aun realizando un estudio histórico que indicara el momento y la forma en que se integraron los elementos culturales impuestos, o especificando cuándo y cómo se dio la integración cultural y cómo se sincretizaron estos cambios en un grupo étnico determinado, seguiría faltando un elemento clave: la identificación de los valores previos a la aculturación. Para poder entender las relaciones cultura-natura se debe, entonces, ahondar en los elementos implícitos — digamos originales- de la simbología cosmogónica de las comunidades analizadas.

Para ello, en continuidad con la discusión que ya hemos abordado, se propone seleccionar algunos de esos valores previos desde el marco de análisis de la naturaleza, la cultura y la identidad (Estrada, 2009), así como desde la ontología analógica propuesta y desarrollada por Descola (2005). Dicho marco derivado de la escuela estructuralista simbólica de Lévi-Strauss $(1995,2009)$ es una aproximación útil para comprender las relaciones cultura-naturaleza existentes en las comunidades tzeltales de los Altos. Con este objetivo se seleccionaron algunas prácticas y saberes relacionados con la cosmovisión tzeltal de la milpa y el maíz.

\section{El análisis de las relaciones cultura-naturaleza desde la ontología analógica}

Explicar las cosmovisiones subyacentes en las relaciones cultura-naturaleza implica deshacerse del repertorio teórico-conceptual de las nociones utilitaristas o productivistas de la naturaleza. Descola $(2003)^{14}$ propone que para analizar la antigua cultura maya es necesario abandonar el determinismo ecológico y examinar las relaciones dialécticas entre cultura y naturaleza. Aquí se plantea que la

\footnotetext{
${ }^{14}$ Ver a Breton, Monod Becquelin y Ruz (2003).
} 
dialéctica mencionada puede entenderse desde las relaciones simbólicas y concepciones que tienen los tzeltales de Los Altos de Chiapas sobre el maíz, como un elemento de intermediación con la naturaleza. ${ }^{15}$

Las relaciones simbólicas y concepciones tzeltales sobre la relación culturanaturaleza se pueden entender conceptualmente desde la ontología analógica. Esta propuesta conceptual desarrollada por Descola proviene de Lévi-Strauss, quién definió que alrededor de la naturaleza gira todo un sistema de ideas, por lo que, aun con características ambientales diferentes, las funciones míticas que construyen los pueblos serán equivalentes y simétricas. Lévi-Strauss (2009) expone que la naturaleza biológica humana condiciona las operaciones intelectuales gracias a las cuales la cultura recibe un contenido empírico. ${ }^{16}$ Por ello argumenta que la prohibición del incesto es la forma de articulación "primaria" en la relación entre la sociedad y la naturaleza que expresa el pasaje del hecho natural al hecho cultural (2009: 66). La relación cultura-naturaleza desde esta perspectiva se basa en "las características objetivas de lo que ofrece la naturaleza y que el espíritu humano va a seleccionar en un contexto cultural dado, a fin de construir conjuntos de significantes" (Descola, 2011: 27). Las condiciones del medio natural son solamente la escenografía que el pensamiento mítico tendrá a bien simbolizar.

La ontología analógica expone las formas simbólicas del imaginario indígena a través de su memoria cosmogónica. Para ello, más adelante se seleccionarán algunos de los elementos simbólicos que se expresan en sistemas de valores, nociones, prácticas, es decir, aspectos o dimensiones del medio social, material y espiritual del saber común que guían las relaciones sociales presentes en las representaciones de las comunidades tzeltales. En este sentido, al igual que Estrada (2009) selecciona los mitos de la tradición oral que narran las cuestiones de animalidad y humanidad ligadas al nahualismo entre los mayas como formas de establecer las fronteras y las continuidades en su cosmogonía, proponemos aquí un análisis de los objetos rituales para explicar una serie de prácticas agrícolas que explicitan las relaciones entre cultura y natura entre los tzeltales alteños de Chiapas.

\section{El altar maya circular}

La cosmogonía (creación del cosmos) sigue estando vigente en muchos de los indígenas mayas alteños, ${ }^{17}$ no solamente tzeltales. Se piensa que el mundo fue

\footnotetext{
15 La propuesta teórica amplia y detallada de dicha ontología se encuentra en el cuarto apartado del Capítulo III, "Les vertiges de l'analogie", del libro Par-delà Nature et Culture (Descola, 2005: 280-321).

16 Algunos de los ejemplos que utiliza para comprender la lógica del sistema de ideas en los estados más primarios del pensamiento humano en su relación con la naturaleza son el miedo a la oscuridad, el instinto de ponerse de pie, el aprendizaje de humanos "animalizados" y algunas cualidades "pre-culturales" entre los primates, como el uso de herramientas (Lévi-Strauss, 2009: 37).

${ }^{17}$ Otros pueblos, como los nahuas, piensan que cada etapa perteneció a una generación humana de diferente hechura (barro, papel, madera, tubérculos), y que la última generación —la actual— está hecha de masa de maíz, por eso, el maíz es su sustento, su carne y su sangre (Florescano, 2000).
} 
creado por el dios Sol en cinco etapas, de las cuales las primeras cuatro fueron sucesivamente destruidas (Florescano, 2000). La composición del cosmos de los mayas coincide esencialmente con la de otras culturas mesoamericanas, como la de los nahuas, en cuanto a que existen cuatro puntos cardinales o rumbos, escenificados con cuatro colores: el este se relaciona con el color rojo; el oeste, con el amarillo; el norte, con el blanco, y el sur (lugar donde moran las almas), con el color negro (Florescano, 2000).

En el caso de los altares tzeltales que se analizaron, se trata de aquellos de forma circular que provienen de la tradición teológica mayanse. ${ }^{18}$ Sin embargo, en los altares circulares la composición del color no coincide con la referida por Thompson (citado en Sotelo, 1988: 11), en la cual el oeste se pone con color negro -en lugar de amarillo- y el sur con amarillo, en vez de negro. El centro del altar, o "corazón", como le llaman los indígenas, se compone de una cruz maya —utilizada como la ceiba conectora de mundos dado que puede ser remplazada por una rama de pino-, un montículo de tierra negra y una tinaja de agua. Estos elementos simbólicos coinciden con lo que Florescano indica como los tres niveles del cosmos maya: el cielo, donde habitan las estrellas, la luna, las constelaciones y el sol; el inframundo, lugar húmedo en donde moran los muertos (noción de alma); y la tierra, lugar de equilibrio entre lo celeste y el inframundo. ${ }^{19}$ Estos tres niveles condensados en la tierra, el agua y la cruz tienen cada uno cuatro esquinas demarcadas con velas de colores, correspondientes al color de los rumbos y que recuerdan las concepciones de "las cuatro esquinas" maya-yucatecas (kan titsikan): "las cuatro esquinas del cielo" (kan tistikab), "las cuatro esquinas de la superficie terrestre" (kan titsiyok 'olkab) y "las cuatro esquinas de la tierra" (kan titsilu'umk), esta última "estructura a la vez los puntos cardinales y los puntos intercardinales, indicados por las cuatro esquinas del altar (kan titsikanché)" (Boccara, 2003: 560). En estos rumbos, el cielo, relata Florescano, es todavía para las culturas indígenas el lugar de residencia de los dioses astrales y de los dioses del viento, el trueno, el relámpago y la lluvia (deidades fecundadoras masculinas). Por su parte, las cuevas son aberturas que comunican al inframundo con la superficie

${ }^{18} \mathrm{~A}$ decir de algunos actores pastorales, el altar maya circular tiene, al menos en la región de los Altos de Chiapas, más de 22 años, lo cual coincide con el proceso de evangelización católica realizado por catequistas, diáconos, sacerdotes, pastores, religiosas y demás servidores indígenas de las comunidades derivado de la Carta Pastoral de 1992 intitulada "500 años sembrando el Evangelio", cuyo objetivo era unificar las prácticas religiosas tradicionales indígenas con las de una religión católica más tolerante a otras formas rituales (Teología India Mayanse..., 1996). Si bien en Chiapas el trabajo pastoral es anterior a la Carta Pastoral de 1992, y se vincula a la iglesia promovida por el Obispo Samuel Ruíz, quien retoma la opción por los pobres derivada del Concilio Vaticano Segundo, tiene su punto más álgido en el Congreso Indígena realizado en 1974 y replicado por la Diócesis en 2013. El trabajo pastoral efectivamente considera al altar maya circular como una práctica apropiada por las comunidades y como una herramienta de evangelización y diálogo que considera elementos de los altares tzeltales ya existentes, como el de muertos, con otros guatemaltecos para reforzar la creencia maya del "corazón del Cielo y corazón de la Tierra" expresada desde la iglesia como la Virgen y Cristo

${ }^{19}$ Sobre este punto se pueden consultar las obras de Ruz $(1998,2006)$. 
terrestre, el lugar donde se albergan las semillas nutricias, regiones oscuras y húmedas (propicias a las deidades femeninas). La montaña, como una representación de la tierra, sigue siendo para los tzeltales un símbolo de la fertilidad.

El Yabwal Balamil simboliza la riqueza agrícola y es representado como un ladino grande y gordo, con los atributos del rayo y la serpiente, dueño de pozos, de los relámpagos y de las nubes, que habita en el interior de la tierra en medio de grandes riquezas: dinero, ganado vacuno, caballos, mulas y pollos. Del interior de la tierra y a través de las cuevas libera las nubes cargadas de agua. Se ostenta como el dueño de los productos de la tierra, de él depende que el maíz viva y fructifique (Florescano, 2000: 19).

Dichos elementos coinciden con el análisis sobre las cuatro esquinas de la milpa y las simbologías de un altar maya circular, el glifo maya del sol y algunos elementos de la filosofía tzeltal relativos al "hombre de cultivo".

Estos paralelos contienen en sí mismos la simbología de lo germinal: la lluvia como semen, las cuevas como vaginas, la montaña como un cuerpo en donde se multiplican los bienes terrenales de la vida (las plantas, las semillas, los seres humanos, los animales) y los elementos sagrados de la naturaleza (tierra, agua, fuego).

En el altar maya circular se agrupan las flores, maíces, calabazas, frutas, chiles, chayotes y demás frutos del trabajo campesino según su tonalidad para demarcar los rumbos. El copal es un elemento central sagrado que se utiliza para orar hacia el altar, existiendo un nivel de intensidad diferente cuando las velas son finalmente encendidas (Figura 1).

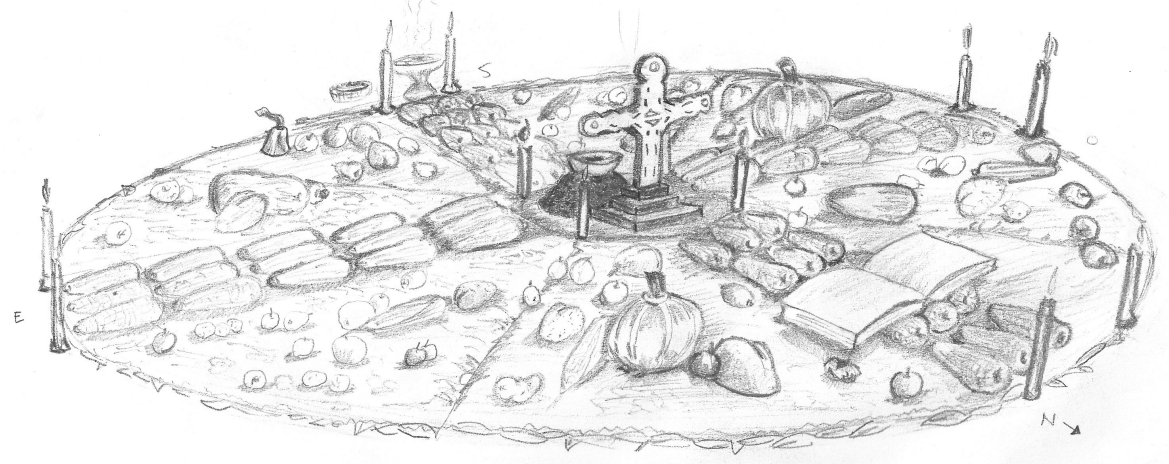

Figura 1. Representación de un altar circular (Dibujo de R. D’Alessandro).

Los elementos de la cosmovisión de Florescano son relativos a los paralelos organizativos de la construcción mítica y simbólica del hombre con su entorno (tiempo y espacio). Asimismo, en el altar se conjugan estas apreciaciones relacionadas con los caminos sagrados de los dioses (dibujados con maíces apuntando en una sola dirección, de norte a sur o de sur a norte), y los caminos de los 
humanos (que igualmente señalan en una sola dirección, de este a oeste). Ambos caminos representan las cuatro esquinas del mundo, y se intersectan en el corazón del altar donde, según un entrevistado tzeltal, "se junta lo sagrado con lo material". Esto último nos habla de que el proceso de significación, ya sea creado o resignificado por la práctica religiosa o proveniente de la teología maya o no, ha sido ya apropiado por los practicantes.

Los altares son considerados en la práctica como una narración que da orden al mito, y que abstractamente podrían ubicarse como el guión y las escenas de la obra simbólica. No narran, sin embargo, la esencia de los personajes, sus elementos de personalidad ni sus dinámicas. De eso se encargará la asignación del alma o ch'ulel, como ha descrito Descola con su categoría de ontología analógica, la que permite analizar los aspectos más íntimos de la asignación cosmológica de los elementos de la naturaleza en su interacción-introyección con lo humano.

\section{El ch'ulel del maíz}

La descripción de los objetos rituales, los mitos y los ritos de los tzeltales muestra las relaciones simbólicas que sostienen con la naturaleza. La representación central que interesa remarcar aquí es la creencia de que el maíz tiene un ch'ulel, o un alma o conciencia que lo conforma como un sujeto/objeto de comunicación con lo sagrado. Esta representación es analizada a partir de la ontología analógica de Descola (2005) para entender el valor simbólico del maíz en las comunidades tzeltales analizadas, y también su relación con formas de conservación que entran en el marco de la significación.

Descola aporta, a partir de lo que él llama la ontología analógica, una serie de criterios teóricos para poder interpretar al ch'ulel de los mayas alteños dentro del complejo mesoamericano. En su tipología, reutiliza la noción de energía como unidad anímica que los humanos pueden extraer simbólicamente de los elementos del medio ambiente para la adquisición de ciertas propiedades mágicas o morales. Esta idea proviene de los trabajos de Viveiros de Castro (1998) sobre el "perspectivismo amerindio", que relata las capacidades de intencionalidad que existen entre humanos y no humanos. Descola retoma estas formas de energía que median entre la naturaleza y la cultura y las disecciona primero en dos: fisicalidad e interioridad. La primera se refiere a la morfología o la forma fisionómica de la energía; mientras que la segunda, a las propiedades internas de dicha energía (alma, mente, consciencia, subjetividades, reflexiones). La fisicalidad y la interioridad son universales y están presentes en toda sociedad, pero se dividen en cuatro formas ontológicas diferentes que contienen todas las propiedades de lo humano y no humano. ${ }^{20}$

\footnotetext{
${ }^{20}$ En el apartado anterior se describen dichas ontologías de Descola. Ésta es la propuesta central de su ya célebre obra Par-delà Nature et Culture (Descola, 2005).
} 
En la ontología analógica, la fisicalidad y la interioridad entre lo no humano y lo humano no son diferentes. Este sistema está basado en la fragmentación y una recombinación de sistemas de clasificación que unifican todas las diferencias o similitudes entre lo humano y lo no humano para utilizarlas en dominios exclusivos tales como la adivinación, la curación de enfermedades, la mala fortuna, etc. Esta ontología es propia de culturas mesoamericanas y busca cumplir una continuidad a partir de ideas de separación o fragmentación de lo humano. Para explicarlo, Descola retoma las referencias de López Austin (1988) sobre la esencia anímica de lo humano en los nahuas: tonacayo, tonalli, teyolia e ihiyotl. Estas formas, también denominadas por Ruz (1998) como los cuatro "alientos de dioses mesoamericanos", tienen, según Descola, capacidades de interioridad que se transmiten o expresan a través de la posesión, la transmigración, la reencarnación, el préstamo y la transformación (nahualismo y tonalismo). ${ }^{21}$

Estas categorías revelan el sentido de interacción cosmológico de la naturaleza en su relación con el mundo social. Una forma de energía útil para describir la cosmovisión de los tzeltales es aquella similar a la del tonalli náhuatl. El tonalli, según López Austin, es una especie de irradiación de calor o luz (parecida a la idea del "aura") que se ubica en la cabeza de los humanos y debe de ser incorporado mediante una ceremonia para que sus portadores tengan vigor y determinación (López Austin, 1988 en Descola, 2005: 291). El tonalli está presente en humanos, pero también en animales y plantas, y puede en casos excepcionales —ebriedad, coito o ensoñación- separarse temporalmente del cuerpo (Descola, 2005: 291).

La referencia simétrica al tonalli náhuatl en las culturas mayas de los Altos de Chiapas es el ch'ulel. ${ }^{22}$ Esta forma anímica, descrita inicialmente por Guiteras (1965), es una especie de "duplicación intangible de las formas y cualidades de las cosas". El ch'ulel tzeltal, según los propios campesinos, es una representación muy cercana a la idea del alma y está presente en todos los seres que crecen en la faz de la madre tierra y en los lugares sagrados: cerros, manantiales y elementos agrícolas.

En el ch'ulel, dice Pitarch, "se aloja en el corazón y ahí residen las emociones, la memoria y el lenguaje $[. .$.$] tiene el mismo perfil que el cuerpo de la persona$ 'pero sin carne, ni huesos', es como una mancha oscura, una sombra espesa" (Pitarch, 1996: 35). El ch'ulel habita simultáneamente en la persona y en el interior de las montañas, donde se resguardan las almas de los niños de los linajes. Los ch'uleles están subordinados a "la gran madre", ${ }^{23}$ muk'me'il (Pitarch, 2003: 606), misma que es conocida en Tenejapa como mux metik y está protegida por los

${ }^{21}$ Para más información veáse Ruz,1998.

${ }^{22}$ El ch'ulel como concepto también existe entre los mayas de tierras bajas, pero es denominado " $i k$ ", la energía vital presente en todo lo vivo. Cuando se habla del $i k$ humano se le denomina pixan (ver Boccara, 2003).

${ }^{23}$ Esta referencia está ligada con la de "madre tierra", "santa tierra", como una divinidad mayor muy presente en el discurso de los tzeltales. 
ajaves (guardianes o espíritus de "seres divinos" ocultos en los lugares sagrados), quienes cuidan de estos sitios y además se encargan de mantener su pureza. Los ajaves protegen al pueblo y a las cosechas. Cuando un humano, en este caso un campesino, necesita recurrir a un ch'ulel determinado para la obtención de un favor (como una buena cosecha) tiene que pedir permiso al ajave para poder conseguir el vigor del ch'ulel. ${ }^{24}$

En la siguiente sección ofrecemos un breve análisis de un altar circular tzeltal que permite remitirnos a nociones de la relación entre naturaleza y cultura desde la ontología analógica: milpa, ch’ulel y maíz.

\section{La milpa, el ch'ulel y el maíz}

En el primer apartado de este artículo se plantea retomar la discusión sobre la posibilidad de utilizar la cosmovisión indígena tzeltal acerca de la milpa y, en especial, en torno al maíz como objeto simbólico, para entender otra forma de relaciones entre la cultura-naturaleza. La relación de la cultura y la naturaleza comenzó en México hace más de ocho mil años; según Bonfil, pacientemente los agricultores contribuyeron a suavizar el perfil del terreno con el cultivo del maíz, lo que ordenó lo que hoy es el territorio nacional desde el nivel del mar hasta tres mil metros de altura mediante "la adecuación reciproca del maíz al hombre y del hombre al maíz” (Bonfil, 1989: 33). Junto con el maíz, se domesticaron otras plantas presentes en la agricultura mesoamericana: frijol, calabaza, chile, maguey, nopal, jitomate, cacao, tabaco, aguacate, algodón, alegría y otras muchas especies vegetales, además de animales como el guajolote, el tepescuintle, etc., así como el control de ciertos productos minerales. El espacio clave para entender el proceso de apropiación del territorio es el manejo en torno al cultivo de la milpa, que, para el caso del sureste, actualmente se realiza por aproximadamente 2.5 millones de campesinos mayas.

Los elementos anteriores sobre la representación de la milpa tzeltal se hacen evidentes a partir del análisis del altar maya circular, donde los cultivos que se ofrendan son propios de la milpa alteña, pero que además tienen la cualidad de poseer ch'ulel. ${ }^{25}$ Entonces nuestra interpretación es que en el altar se representan como producto del trabajo de la milpa aquellos que tienen ch'ulel: chayote, chile, jícama, calabacitas, calabaza, yuca, camotes, pepinos, semillas de girasol, cacahuates, cueza, haba, chícharos, etc. ${ }^{26}$ La milpa se representa en el altar maya no sólo como el jardín de cultivo de plantas “originales o verdaderas", sino como una forma de génesis de la vida, o un espacio dotador ${ }^{27}$ y negador de ch'ulel.

\footnotetext{
${ }^{24}$ Entrevista a bakilal de Tenejapa, SCLC, Chiapas, 30 de enero de 2014.

25 Conversación con R. Mariaca, SCLC, Chiapas, febrero de 2014.

${ }^{26}$ Sobre la alimentación proveniente de la milpa, ver Ruz (1987).

27 Los animales e insectos de la milpa también tienen ch'ulel: jabalí, gallina, novillos, guajolotes, ratas, venados, larvas, orugas, caracoles.
} 
Esta variedad de ch'uleles (que tienen otras 13 formas particulares denominadas labs) aporta elementos para entender por qué la milpa es utilizada como representación de la naturaleza y espacio de comprensión mítica. Según Gómez (1997), quien trabajó con comunidades tzeltales de Tenejapa, el ethos maya es un sistema en donde el valor central es la capacidad de una persona para el aprendizaje (observación, imitación, reiteración) y para la obtención de sabiduría, que le atraerá prestigio y poder. Pero el poder de la sabiduría en el mundo tzeltal no es relativo al éxito económico, sino que conlleva un conocimiento profundo de la milpa para llegar a ser una "persona de cultivo". Se tienen entonces cuatro elementos, como cuatro esquinas, para el ser completo: aprendizaje, sabiduría, prestigio y poder.

$\mathrm{Al}$ analizar el altar maya circular, ${ }^{28}$ nos damos cuenta de que la división en cuatro puntos cardinales coincide con la idea de los cuatro colores (aunque la dirección de los colores sea diferente), también destacan el maíz y los cultivos de la milpa como objetos simbólicos y componentes centrales. Asimismo, en el altar se expresan elementos teológicos (como la idea de la madre tierra, del centro, de los rumbos y niveles, de las interacciones entre lo sagrado y lo material), pero también implícitamente ciertos valores como la equidad, dado que todos los lados del altar tienen el mismo volumen de alimentos; centralidad, en cuanto a que el centro se distingue como el punto de paso de niveles o mundos, y como la intersección entre los humanos y los dioses; la diversidad se expresa no solamente en los colores, sino en la variedad de cultivos que componen la ofrenda; horizontalidad, en cuanto a que todos los rumbos son igualmente importantes, y el trabajo, como la fuerza que permite que siga existiendo la vida y los frutos de la madre naturaleza. Como se observa en el dibujo del altar (Figura 1), los maíces llevan una dirección de norte a sur y de este a oeste. Según se comenta, el camino de norte a sur es transitado por los dioses y el de este a oeste por humanos. La intersección de los caminos y direcciones marcadas en el altar con maíces simbolizan el encuentro divino de dios con lo humano, ${ }^{29}$ lo que se asemeja a la creación del hombre y con la idea del movimiento descrita en los mitos del Paxil retomados del Popol Vuh. Ahí se habla de la creación del hombre con masa de maíz de la diversidad de esta planta: "Los animales (...) les dieron noticia (a los dioses creadores del hombre) de las mazorcas amarillas, y las mazorcas blancas, les dijeron que fueran a Paxil (...) y les enseñaron el camino". ${ }^{30}$ Además de tal variedad se aprecia la idea de que el grano es rescatado, encontrado, transportado, lo que refiere movilidad y dispersión: ${ }^{31}$ "el maíz

\footnotetext{
${ }^{28}$ Se requiere de una mayor profundización en momentos rituales para poder establecer como homogéneas las observaciones aquí presentadas. En 2016 se realizaron nuevas inmersiones de campo para recopilar mayor información sobre ese punto, pero se encontraron símiles con otros altares tzotziles sin lograr hacer una diferenciación específica entre ambos.

${ }^{29}$ Charla informal (sin precisión del lugar).

${ }^{30}$ Tomado del análisis del Popol Vuh por A. Recinos (1947) en Navarrete, 2002: 15.

${ }^{31}$ No se olvide que la hipótesis tripartita del maíz sugiere que los centros de domesticación ocurrieron en diferentes lugares, lo cual confirma el núcleo del mito. Sin movilidad y sin dispersión no existirían las 59 razas de maíz reconocidas en México.
} 
estaba dentro de la panza de un coyote descuartizado, y que un pequeño gavilán lo trajo junto con sangre del mar para hacer su masa". ${ }^{32}$ Igualmente, entre los k'iche's de Guatemala existe la creencia de que un cuervo dejó caer una mazorca que un joven recogió, al probarla decidió seguir al cuervo y las llevó a su pueblo (Navarrete, 2002: 29).

Entre los tzeltales hay historias que hablan de una hormiga arriera que le quitó el maíz a los humanos y lo llevó a una montaña porque estos no lo cuidaban. ${ }^{33}$ Este mito refiere también a la idea de un dios padre "dueño de la hormiga" que le ordenaba a ésta traer el maíz. Tal creencia se ha sincretizado con el cristianismo, en donde el dueño es ahora Jesucristo, y en lugar de una hormiga se habla de un abejorro, que no va a llevar el maíz sino a rescatarlo del resguardo de Jesucristo (Shaw en Navarrete, 2002). En todo caso, tanto la hormiga como el abejorro son castigados por el "dueño", que les amarra la cintura.

\section{Circularidad y el ciclo de la vida y aprendizaje}

La forma circular y cuatripartita del altar es parecida a la del glifo k'in (Figura 2) que significa "día-sol". Según Boccara, la medida de las unidades agrícolas se apoya en la cifra cuatro, en referencia a los cuatro puntos cardinales: "El cuatro está asociado a la milpa, al cielo y al padre cósmico (el sol), y también al varón u hombre maya (...) a las cuatro esquinas de la milpa y también a las cuatro esquinas del cielo y la tierra" (2003: 560-561).

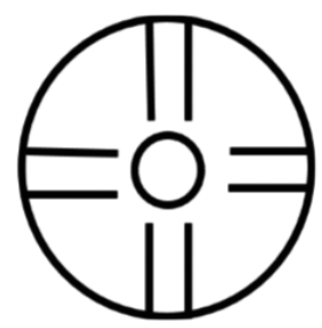

Figura 2. Glifo maya k’in (según Boccara, 2003, dibujo de R. D’Alesandro).

Como bien lo indica Gómez (1997), la milpa es un espacio en donde se vive la experiencia del aprendizaje y se ejercita un proceso de reflexión y autoaprendizaje ligado al contacto con la naturaleza. Las nociones de las cuatro esquinas de la milpa, junto con los cuatro elementos de "la persona del cultivo" que proponemos basados en Gómez, infieren una similitud entre el hombre y la milpa. Sin embargo, falta agregar que la noción de las cuatro esquinas contiene un simbolismo

\footnotetext{
${ }^{32}$ Memorial del Sololá de tradiciones del pueblo k'acchiquel (Navarrete, 2002: 15).

${ }^{33}$ Conversación con un bankilal fuera de la iglesia de San Ildefonso, Tenejapa (2013).
} 
fundamental en el plano de los cuatro puntos cardinales: el centro como unidad primordial. Sobre esto encontramos otra similitud interesante descrita por De la Garza (1975), quien retoma las descripciones del fray Diego de Landa sobre el centro como eje cósmico representado por la ceiba, donde se aprecia una noción, que bien puede ser asociada con la milpa, en el sentido de que en ella "abundan alimentos (...) y es el lugar por excelencia de la fertilidad de la tierra (...) el sitio de energía de la vida" (De la Garza en Sotelo, 1988: 51). Desde esta perspectiva el punto central es simbolizado en los altares circulares mediante la cruz maya. Ahí se expresa la vida misma del hombre y del universo. Nos atrevemos a proponer que milpa y hombre - centro cósmico de sus planos cardinales - conforman un solo elemento.

La milpa es un espacio de reproducción del microcosmos simbólico. Cada etapa del ciclo agrícola dentro del calendario maya, desde la siembra hasta la cosecha, está acompañada por ritos y mitos. Valdría referirnos a un mito conocido relacionado con la práctica de selección de semilla en la milpa. Según la narración de uno de los entrevistados:

[...] cuando se desgrana la mazorca del maíz para la selección de semilla en la siembra, esto debe hacerse solamente de la parte media de la mazorca [...] Hay que dejar fuera - dice- todas las semillas que provienen "de la cola" y "de la cabeza" de la mazorca. Si estos granos restantes - los que se quedan en las mazorcas- no se utilizan para alimentarse — para hacer tortilla o pozol— sucederá que todas las semillas sembradas serán comidas por "el mapache" que vendrá de noche y escarbará para comerse las semillas. Si los granos sobrantes en la mazorca son dados a los pollos o a los animales el maíz, vendrá el mapache de igual manera. Si por el contrario - y es la única manera de evitarlo— la familia se come las semillas sobrantes en la mazorca, no vendrá el mapache. ${ }^{34}$

El mapache representa aquellas fuerzas de la naturaleza o exteriores que pueden afectar el mundo social. Es una fuerza simétrica a la del tejón, analizado por Julieta Valle (2003) en "la fiesta de los elotes" de los nahuas de Chicontepec y también de los tepehuas de Tlachichilco. En ambos casos se narra cómo:

[...] la naturaleza incursiona en el ámbito de lo social a través de individuos disfrazados de tejón, quienes intentan comerse los elotes recién cosechados. Su transgresión representada en diferentes tentativas de robo, da pie a un juego en el que otros personajes regañan a uno de ellos y lo sacan; el hombre-tejón vuelve a entrar, se esconde, está a punto de salirse con la suya. Al final, los otros lo atrapan, lo colocan en un horcón, le amarran los pies y las manos a un palo y terminan por tirarlo al monte. De manera ejemplar, se devuelve a la naturaleza, que es su ámbito. No tiene nada que hacer en el mundo social ni por qué alimentarse de los elotes, fruto preciado del trabajo humano (Valle, 2003: 236)

\footnotetext{
${ }^{34}$ Esta práctica se realiza en el mes de marzo durante la siembra. La narración del mito proviene de uno de los informantes clave. Entrevistas realizadas el 3 y 9 de marzo de 2013.
} 
Este mito conlleva a reflexionar que la milpa no es solamente un espacio de representación cultural de las relaciones sociedad-naturaleza, sino de una "fricción heurística que permite diseñar formas de conexión muy diversas" (Hanks, 2003: 162) para aprender de la naturaleza y aprehender lo natural. Es al mismo tiempo un espacio de aprendizaje sobre uno mismo y del sistema ceremonial y cosmogónico que acompaña la producción, un espectro de múltiples configuraciones que nos hablan de lo tzeltal u "hombre verdadero" y de algunas de sus características: fuerza para participar en el trabajo, sabiduría para conocer la naturaleza, espiritualidad para comprender y ofrendar correctamente los productos de la cosecha y la capacidad de "crear vida".

Según Boccara, "cultivar maíz es una operación de vida, homóloga a la de hacer un niño [...] Se crea una nueva vida que tiene su origen en un espacio mítico" (2003: 547). La idealización del maíz como un ente humanizado se representa en la creencia de que la planta escucha lo que los humanos le dicen. Como ya se dijo, se considera que todo el maíz ${ }^{35}$ (así como el frijol y la calabaza) —independientemente del color- tiene ch'ulel.

Para explicar una de las cualidades humanas del maíz, tomemos esta narración de Guiteras sobre la siembra: "Cuando se siembra se hacen tortillitas de $2.5 \mathrm{~cm}$, para ponerlas junto con las semillas de maíz. Esto asegura que el maíz siga a la tortilla y salga" (1965: 43). Esta práctica le da un sentido humano (y animal) al maíz al salir de la tierra en búsqueda de alimento. El maíz encontrará la salida al mundo subiendo por la tierra para alimentarse de su tortillita.

Otro ejemplo referente a la interioridad humana del maíz es que éste y los frijoles tienen un referente con figuras principales de la religión católica:36 "el maíz como cristo rey y los frijoles como el espíritu santo. Asimismo, otras plantas como los chayotes y los chilacayotes son entidades relacionadas con la imagen de la virgen de Guadalupe". ${ }^{37}$ La idea coincide con el término náhuatl tonacayo, que designa "la sustancia real que forma una totalidad discreta presente tanto en humanos como en plantas, donde el maíz es el jefe principal" (López Austin en Descola, 2005: 290).

\footnotetext{
${ }^{35}$ No todos los maíces tienen ch'ulel, en alguna ocasión escuché traducir lo que significa un maíz transgénico como "maíz sin ch'ulel".

${ }^{36}$ El catolicismo tradicional adopta creencias autóctonas sincréticamente. Su principal característica no es el sincretismo, sino que quienes administran los ritos son figuras y principales tradicionales que no son designadas por el episcopado. El caso más conocido en Chiapas es el de la comunidad tzotzil de San Juan Chamula vigente también para Tenejapa.

${ }^{37}$ Conversación informal con Ramón Mariaca, estudioso de los simbolismos de la milpa maya, que repite la narración de campesinos tzotziles sobre la existencia de "maíces-padre", "maíces-madre" y "maíces-hijo". Asimismo, narra haber escuchado de la existencia de "maíces-corazón" y de "maíces-policía". Estas diferentes representaciones de maíces parecen guardar la esencia del sincretismo cristiano de la tríada "hombre-dios-espíritu santo" y de la noción de "protección", tan propia de la cosmovisión maya: El maíz-corazón para la protección familiar y maíz-policía para cuidar la milpa, SCLC, Chiapas, 20 de febrero de 2014.
} 
Analizar un altar desde una forma teórico-conceptual ligada al modelo de aculturación y utilitarismo ecológico no hubiera permitido abordar la amplitud simbólica de los modos de apropiación social de la naturaleza desde la cosmovisión tzeltal. De allí la utilidad del análisis con elementos del estructuralismo simbólico. Se ha analizado solamente una manifestación de las concepciones anímicas que giran en torno a la milpa y al maíz, a partir de la descripción no exhaustiva de un altar circular. ${ }^{38}$ Existen, sin embargo, innumerables prácticas, saberes y mitos ligados a la milpa tzeltal alteña que revelan todo un sistema complejo de fisicalidades e interioridades (humanas y de la naturaleza) que siguen perdurando a pesar de los procesos de aculturación o control cultural en la agricultura campesina.

Retomamos aquí algunas de las aportaciones al respecto: ${ }^{39}$ 1) la práctica de oraciones para realizar la siembra y para el cultivo; 2 ) la construcción de las casas en el centro de los terrenos, y no en los costados; 3 ) la utilización de objetos rituales (copal, tabaco "bobo" y últimamente cigarros) y bebidas (posh) para la protección de la siembra, o para la maldición de otras milpas; 4) la dirección de las plegarias hacia el suelo (inframundo en la cosmovisión tzeltal); 5) la realización de las ceremonias en la milpa; 6) la utilización del ciclo lunar para la planeación agrícola; 7) la utilización del calendario maya de 18 meses con 20 días para la producción; 8) la planificación agrícola a través de los sueños; 9) las formas de predicción del tiempo; 10) la utilización de insectos o animales en la predicción de la lluvias; 11) la ubicación "aérea" de los maíces seleccionados como semillas para la siembra, y 12) la sacralización de la naturaleza y del maíz (tierra, agua, fuego) y de ciertos lugares (cuevas, montañas, manantiales).

Hablemos de la última, reconociendo la sacralización del maíz rojo como una medicina utilizada en algunos rituales de sanación. Cuando un campesino encuentra una mazorca roja, le da un sentido de sagrada. Antes de describirlo, comentaremos el mito que explica la existencia del maíz rojo: un hombre perezoso se casa con una mujer (representada a veces como la hija de un ángel, o como una virgen, o como la ch'umeltik, "la madre de todos", o la luna). Al regresar a su casa un día, el hombre no encuentra la comida hecha y ve que la mujer está todavía desgranando el maíz, por lo que, enojado, la golpea en la nariz. Ella comenzó a sangrar sobre el maíz que desgranaba y el maíz se tiñó de rojo. ${ }^{40}$

En la narración, la mujer no es cualquier mujer, sino la hija de un ángel que tiene un origen sagrado. El componente místico se expresa también en la aparición casual de los maíces rojos (generalmente uno o dos por milpa). Esto se

${ }^{38}$ El altar circular responde a una intersección de lo teológico en lo ritual, es decir, se impone como una forma ritual que toma elementos de la ritualidad cotidiana y sincretizada de los pueblos, para establecer nuevos elementos. Mientras que el altar recto de los tzeltales es parte de su práctica cotidiana, el altar circular se constituye como un medio de interacción entre los grupos locales y los interventores.

${ }^{39}$ La mayoría de ellas enriquecidas o retomadas de Mariaca (2003).

${ }^{40}$ Entrevistas P.G. (mayo 2012), J. G. (enero 2013) y A. G. (mayo 2013). 
debe a que el maíz rojo es una variación genética del maíz amarillo, por lo que los campesinos que siembran semilla roja obtienen mazorcas amarillas. He aquí la utilización del maíz rojo para sanación:

(...) cuando tenía 7 años se enfermó. La causa de la enfermedad fue una caída la cual le provocó que padeciera del J'ilel (pérdida, encierro o movimiento del ch'ulel a algún lugar indebido por un fuerte golpe). Para curarse, su padre lo llevó con un poxtabané. El sanador era una persona muy reconocida que sabe curar el j’ilel. Sacó una jícara en la que puso una mazorca de maíz rojo y lo desgranó mezclándolo con agua (se cree que el sólo contacto del maíz rojo con el agua la convierte en agua bendita). Retiró los granos del maíz, tomó el agua con la boca y comenzó a rociarla por todo el cuerpo especialmente en el lugar en donde se había golpeado. Después utilizó una planta con hojas pequeñas (paita), y comenzó a golpear al niño al modo de una limpia” (D’Alessandro, 2014: 249).

Aparentemente, el aspecto mágico del maíz se pierde una vez desgranada la mazorca. Se desconoce la utilidad que se le da a los granos después de utilizarlos en la sanación de un mal. Habitualmente las ramas con que se "ramea" a alguien son desechadas. Se tiene la creencia de que el contacto de estas plantas con la enfermedad hace que la planta absorba el mal.

\section{Consideraciones finales}

Existen elementos suficientes para describir la existencia de vínculos cosmogónicos entre las formas culturales y de agrupación social de los indígenas tzeltales y su control sobre los recursos naturales accesibles. El objetivo de este artículo ha sido ahondar sobre las concepciones de los mayas tzeltales de Los Altos de Chiapas en relación con la naturaleza y el maíz. La hipótesis que propone al maíz como una planta-objeto cultural, en la que los tzeltales expresan su cosmovisión sobre la naturaleza, se apoya en la posibilidad de identificar las fisicalidades e interioridades en la milpa. Ello resulta evidente a partir del análisis de los elementos ceremoniales y de prácticas y creencias que revelan valores o sentidos simbólicos explícitos y coherentes con su propia cosmovisión.

La discusión que propone Estrada y su actualización desde las aportaciones teóricas de Descola resultan útiles para entender la humanización del maíz dentro de la cosmovisión maya. La propuesta ontológica analógica de Descola para describir las relaciones naturaleza-cultura sirve como un marco actualizado en el que pueden incluirse las prácticas agrícolas y los símbolos que se han analizado. Al construir la representación de la naturaleza en un objeto ritual (el altar circular) se obtiene una representación complementaria a los mitos, la cual permite observar las cualidades sobrenaturales de los vegetales a través de su ch'ulel.

El presente texto fortalece la idea de que la construcción social y de sentido identitario tzeltal a través de la práctica agrícola que se expone mediante la idea 
del hombre sabio o "de cultivo" como cuidador y generador de abundancia, una aportación que debe incluirse en las representaciones de la naturaleza. En este sentido, lo humano juega un rol totalmente diferente al asignado por el dualismo etnocéntrico y occidental que separa a la cultura de la naturaleza. La integración de lo humano dentro del cosmos mediante esquemas pragmáticos del saber, la práctica, la reproducción de lo vivo y las representaciones simbólicas de la naturaleza continúa existiendo y practicándose en la cultura maya tzeltal de Tenejapa.

\section{BIBLIOGRAFÍA}

Arhem, Kaj

2001 "La red cósmica de la alimentación. La interconexión de humanos y naturaleza en el noroeste de la Amazonia”, Naturaleza y sociedad. Perspectivas antropológicas, pp. 214-236, P. Descola y G. Pálsson (eds.). México: Siglo XXI.

Barrera-Bassols, Narciso y Víctor Toledo

2005 "Ethnoecology of the Yucatec Maya: Simbolism, Knowledge and Management of Natural Resources", Journal of Latin American Geography, 4(1): 9-41. DOI: 10.1353/lag.2005.0021.

Bastide, Roger

1976 El sueño, el trance y la locura. Buenos Aires: Amorrortu.

Boccara, Michel

2003 “Vivir es hacer. Volverse ‘viantepasado' o el dominio del espacio transicional”, Los espacios mayas: representaciones, pp. 533-576, Alain Breton, Aurore Monod Becquelin y Mario H. Ruz (eds.). México: Universidad Nacional Autónoma de México, Instituto de Investigaciones Filológicas, Centro de Estudios Mayas y Centro de Estudios Mexicanos y Centroamericanos.

Boege, Eckart

2009 "Centros de origen, pueblos indígenas y diversificación del maíz", Ciencias, 93 (octubre-marzo): 18-28.

Broda, Johanna

1991 "Cosmovisión y observación de la naturaleza: El ejemplo del culto a los cerros", Arqueoastronomía y etnoastronomía en Mesoamérica, pp. 461-500, Johanna Broda, Stanislaw Iwaniszewski y Lucrecia Maupomé (eds.). México: Universidad Nacional Autónoma de México, Instituto de Investigaciones Históricas.

Bonfil Batalla, Guillermo

1989 La teoría del control cultural en el estudio de los procesos étnicos. México: Centro de Investigaciones y Estudios Superiores en Antropología Social.

2005 México Profundo. México: Debolsillo. 
Breton, A., Aurore Monod Becquelin y Mario H. Ruz (eds.)

2003 Los espacios mayas: representaciones, utilizaciones y creencias. México- París: Universidad Nacional Autónoma de México, Instituto de Investigaciones Filológicas, Centro de Estudios Mayas y Centro de Estudios Mexicanos y Centroamericanos.

Chamoux, Marie

1994 "La difusión de tecnologías entre los indígenas de México: una interpretación”, Semillas de industria, pp. 123-144, Mario Humberto Ruz (ed.). México: Centro de Investigaciones y Estudios Superiores en Antropología Social.

D’Alessandro, Renzo

2014 Formes sociales de conservation du maïs dans l'agriculture txeltal de Tenejapa, Chiapas, Mexique, tesis de Doctorado en Sociología. Montpellier: Université Paul-Valéry Montpellier III.

Descola, Philippe

2003 "El paisaje maya y su historia. Comentario", Los espacios mayas: representaciones, pp. 199-208, A. Breton, A. Monod Becquelin y Mario H. Ruz (eds.) México: Universidad Nacional Autónoma de México, Instituto de Investigaciones Filológicas, Centro de Estudios Mayas y Centro de Estudios Mexicanos y Centroamericanos.

2005 Par-delà nature et culture. Paris: Gallimard.

2011 L'écologie des autres. L'anthropologie et la question de la nature. Paris: Quae (Collection Sciences en Questions).

Descola, Philippe y Gísli Pálsson

2001 Naturaleza y sociedad. Perspectivas antropológicas. México: Siglo XXI.

Estrada Ochoa, Adriana

2009 "Naturaleza, cultura e identidad. Reflexiones desde la tradición oral maya contemporánea", Estudios de Cultura Maya, XXXIV: 181-201. DOI: http:// dx.doi.org/10.19130/iifl.ecm.2009.34.35.

Ellis, Erle C.

2011 "Anthropogenic Transformation of the Terrestrial Biosphere", Proceedings of the Royal Society A: Mathematical, Physical and Engineering Science, 369: 10101035. DOI: $10.1098 /$ rsta.2010.0331.

Ellis, Erle C., Erika C. Antill y Holger Kreft

2012 "All Is Not Loss: Plant Biodiversity in the Anthropocene", PLOS ONE 7(1): e30535. DOI:10.1371/journal.pone.0030535.

Ellison, Nicolas

2013 Semé sans compter. Paris: Éditions de la Maison des Sciences de l'Homme.

Florescano, Enrique

2000 "La visión del cosmos de los indígenas actuales", Desacatos. Saberes y Razones, 5: 15-29. 
Ford, Anabel y Ronald Nigh

2015 Maya Forest Garden: Eight Millennia of Sustainable Cultivation of the Tropical Woodlands. Walnuk Creek: Left Coast Press Inc.

Gadgil, Madhav y Ramachandra Guha

1992 This Fissured Land: An Ecological History of India. Berkeley: University of California Press.

Garza, Mercedes de la

1975 El hombre en el pensamiento religioso náhuatl y maya. México: Universidad Nacional Autónoma de México.

1984 El universo sagrado de la serpiente entre los mayas. México: Universidad Nacional Autónoma de México, Instituto de Investigaciones Filológicas, Centro de Estudios Mayas.

2012 Sueño y alucinación en el mundo náhuatl y maya. México: Fondo de Cultura Económica y Universidad Nacional Autónoma de México, Instituto de Investigaciones Filológicas, Centro de Estudios Mayas.

Godelier, Maurice

1984 Lo ideal y lo material. México: Taurus (Colección Humanidades).

Gómez Muñoz, Maritza

1997 "Los aprendizajes comunitarios en los Altos de Chiapas", Indígenas en la Escuela, pp. 53-84, María Bertely Busquets y Adriana Robles Valle (coords.). México: Grupo Ideograma Editores.

Guiteras Holmes, Calixta

1965 Los peligros del alma: Visión del mundo de un tsotzil. México: Fondo de Cultura Económica.

Hanks, William

2003 "Reduction and the remaking of the social landscape in colonial Yucatán", Los espacios mayas: representaciones, pp. 161-180, Alain Breton, Aurore Monod Becquelin y Mario H. Ruz (eds.). México: Universidad Nacional Autónoma de México, Instituto de Investigaciones Filológicas, Centro de Estudios Mayas y Centro de Estudios Mexicanos y Centroamericanos.

Harris, Marvin

2003 Introducción a la antropología general, 6a. edición. Madrid: Alianza Editorial.

Hermitte, Esther

1970 Control social y poder sobrenatural en un pueblo maya contemporáneo. México: Instituto Indigenista Interamericano.

Hobbs, Richard J., Eric Higgs y James A.Harris

2009 "Novel Ecosystems: Implications for Conservation and Restoration", Trends in Ecology \& Evolution, 24: 599-605. http://dx.doi.org/10.1016/j. tree.2009.05.012. 
Holland, William Roland

1963 Medicina maya en Los Altos de Chiapas. México: Instituto Nacional Indigenista.

Jacorzynski, Witold

2004 Entre los sueños de la razón. Filosofía y antropología de las relaciones entre el hombre y el ambiente. México: Centro de Investigaciones y Estudios Superiores en Antropología Social.

Köhler, Ulrich

1995 Chonbilal Ch'ulelal-Alma Vendida. Elementos fundamentales de la cosmología y religión mesoamericana en una oración en maya-tzotzil México: Universidad Nacional Autónoma de México.

Lapierre, J. William

1968 Essai sur le fondement du pouvoir politique. Gap: Éditions Ophrys (Publications de la Faculté des Letres d'Aix-en-Provence).

Leclerc, Christian y Geo Coppens d' Eeckenbrugge

2011 "Social Organization of Crop Genetic Diversity. The $G \times E \times S$ Interaction Model", Diversity, (diciembre) 4 (1): 1-32.

Lévi-Strauss, Claude

1995 Antropología estructural. Barcelona: Paidos.

2009 Las estructuras elementales del parentesco. Barcelona: Paidós.

López Austin, Alfredo

1988 The Human Body and Ideology. Concepts of the Ancient Nahuas. Salt Lake City: University of Utah.

2001 "El núcleo duro de la cosmovisión y la tradición mesoamericana", Cosmovisión, ritual e identidad de los pueblos indígenas de México, pp. 47-65, Johanna Broda y Félix Báez (eds.). México: Fondo de Cultura Económica.

2003 "Cuatro mitos mesoamericanos del maíz", Sin maíz no hay país, pp. 29-35, G. Esteva y C. Marielle (coords.). México: Consejo Nacional para la Cultura y las Artes, Museo de las Culturas Populares.

Mariaca Méndez, Ramón

2003 "Prácticas, decisiones y creencias agrícolas mágico-religiosas presentes en el sureste de México”, Etnobiología, 3: 66-78.

Medina, Andrés

1991 Tenejapa: Familia y tradición en un pueblo tzeltal. Tuxtla Gutiérrez: Gobierno Estatal de Chiapas e Instituto Chiapaneco de Cultura.

Navarrete Cáceres, Carlos

2002 Relatos mayas de tierras altas sobre el origen del maíz: Los caminos del Paxil. México: Universidad Nacional Autónoma de México, Instituto de Investigaciones Antropológicas (Colección Textos. Serie Antropología e Historia Antigua de México, 3). 
Page Pliego, Jaime Tomás

2005 El mandato de los dioses. Etnomedicina entre los tzotziles de Chamula y Chenalhó. México: Universidad Nacional Autónoma de México, Instituto de Investigaciones Antropológicas, Programa de Investigaciones Multidisciplinarias sobre Mesoamérica y el Sureste.

Pitarch, Pedro

1996 Etnografía de las almas tzeltales. México: Fondo de Cultura Económica.

2003 "Dos puntos de vista, una sola persona: El espacio en una montaña de almas" Los espacios mayas: representaciones, pp. 603-612, Alain Breton, Aurore Monod Becquelin y Mario H. Ruz (eds.). México: Universidad Nacional Autónoma de México, Instituto de Investigaciones Filológicas, Centro de Estudios Mayas y Centro de Estudios Mexicanos y Centroamericanos.

Pitarch, Pedro, Jerôme Baschet y Mario H. Ruz (eds.)

1999 Encuentros de almas y cuerpos entre Europa medieval y el mundo mesoamericano. Tuxtla Gutiérrez: Universidad Autónoma de Chiapas, Instituto de Estudios Indígenas.

Pozas Arciniega, Ricardo

1977 Chamula: Un pueblo indio de los altos de Chiapas. México: Instituto Nacional Indigenista.

Ruz, Mario Humberto

1987 "La alimentación de los grupos mayas", Cuadernos de Nutrición, 10 (3): 3-15.

1994 Semillas de industria. México: Centro de Investigaciones y Estudios Superiores en Antropología Social.

1998 "Los cuatro rumbos del Cielo", Diálogo entre Fronteras, 10: 7-12.

2004 "Átomos de fuego europeos, alientos de dioses mesoamericanos", Cuerpo humano e imagen corporal. Notas para una antropología de la corporeidad, pp. 15-21, Carlos Aguado Vázquez (ed.). México: Universidad Nacional Autónoma de México, Instituto de Investigaciones Antropológicas

2006 "Un tissu de dieux. Croyances et dévotions mayas à l'époque coloniale", Religions et Histoire, (marzo-abril) 7: 72-77.

Sotelo Santos, Laura Elena

1988 Las ideas cosmológicas mayas en el siglo XVI. México: Universidad Nacional Autónoma de México, Instituto de Investigaciones Filológicas, Centro de Estudios Mayas.

Steward, Julian H. y Demitri B. Shimkin

1961 "Some Mechanisms of Sociocultural Evolution”, Daedalus, Evolution and Man's Progress, 90 (3): 477-497.

Teología india mayanse. Memorias, experiencias y reflexiones de encuentros teológicos regionales 1996 Guatemala: Centro Nacional de Ayuda a las Misiones Indígenas 
Toledo, Víctor Manuel

1996 "Saberes indígenas y modernización en América Latina: Historia de una ignomia tropical”, Etnoecológica, II (4-5): 135-147.

Valle, Julieta

2003 "Reciprocidad, jerarquía y comunidad en la tierra del trueno (la Huasteca)", La comunidad sin límites. Estructura social y organización comunitaria en las regiones indígenas de México II, 211-340, Saúl Millán y Julieta Valle (coords.). México: Instituto Nacional de Antropología e Historia (Colección Etnografía de los Pueblos Indígenas de México).

Villa Rojas, Alfonso

2003[1968] "Apéndice I. Los conceptos de espacio y tiempo entre los grupos mayances contemporáneos", Tiempo y realidad en el pensamiento maya. Ensayo de acercamiento, 4a ed., pp. 123-168, Miguel León Portilla (ed.), Eric S. Thompson (pról.). México: Universidad Nacional Autónoma de México, Instituto de Investigaciones Históricas (Serie Culturas Mesoamericanas, 2).

Viveiros de Castro, Eduardo

1998 "Cosmological Deixis and Amerindian Perpectivism”, The Journal of the Royal Antropological Institute, 4: 469-488. DOI: 10.2307/3034157.

Vogt, Evon Z.

1966 Los zinacantecos. México: Instituto Nacional Indigenista.

1979 Ofrendas para los dioses. México: Fondo de Cultura Económica. 
